\title{
Recombinantly Expressed MeICT, a new Toxin from Mesobuthus Eupeus Scorpion, Inhibits Glioma Cell Proliferation and Downregulates Annexin A2 and FOXM1 Genes
}

Hoda Ayat ( $\square$ hoda.ayat@gmail.com )

Shahrekord University https://orcid.org/0000-0001-5699-3994

Maryam Shahbazi Gandomkari

Shahrekord University

Ali Mohammad Ahadi

Shahrekord University

\section{Research Article}

Keywords: Recombinant MelCT peptide, Glioma cells, Annexin A2, FOXM1, MMP-2

Posted Date: November 22nd, 2021

DOI: https://doi.org/10.21203/rs.3.rs-1010027/v1

License: (c) (1) This work is licensed under a Creative Commons Attribution 4.0 International License.

Read Full License 


\section{Abstract}

Gliomas are highly invasive and lethal malignancy that do not respond to current therapeutic approaches. Novel therapeutic agents are required to target molecular mechanisms involved in glioma progression. MelCT is a new short-chain toxin isolated from Mesobuthus eupeus scorpion venom. This toxin contained 34 amino acid residues and belongs to chloride channels toxins. In this study, the coding sequence of MeICT was cloned into the pET32Rh vector and a high yield of soluble recombinant MelCT was expressed and purified. Recombinant MeICT-His significantly inhibited the proliferation and migration of glioma cells at low concentration. In vivo studies showed that MeICT was not toxic when administrated to mice at high doses. We also determined the effect of MelCT on the mRNA expression of MMP-2, Annexin A2 and FOXM-2 that are key molecules in the progression and invasion of glioma. Expression of Annexin A2 and FOXM1 mRNA was significantly down-regulated following treatment with MelCT. However, no significant decrease in the expression of MMP-2 gene was identified. In this study a short toxin with four disulfide bonds was successfully produced and its anti-cancer effects was detected. Our findings suggest that recombinant MeICT can be considered as a new potent agent for glioma targeting.

\section{Introduction}

Malignant glioma is a common primary brain tumor and is considered among the most lethal cancers (Liang et al., 2020). The tumor is highly invasive with fast cell proliferation and unusual tendency to spread to healthy brain tissue. Glioma cells spread through two main mechanisms: degradation of the extracellular matrix (ECM) by matrix metalloproteinases (MMPs) such as MMP-2 and deformation of the cell shape via ionic gradients that occurred by overexpression of ion channels such as chloride $\left(\mathrm{Cl}^{-}\right)$ channels (Turner and Sontheimer, 2014; Dardevet et al., 2015). The search for therapeutic agents that target these molecular mechanisms without affecting normal tissues is increasingly being considered.

MelCT, a novel short-chain toxin, was isolated from Iranian Mesobuthus eupeus scorpion in our laboratory (llkhanizade et al., 2011). MelCT contains 34 amino acids crosslinked by four disulfide bridges and displays $81 \%$ sequence similarity with chlorotoxin (CTX). CTX from the venom of Leiurus quinquestriatus scorpion contains 36 amino acids and a compact structure of an $\alpha$-helix and three $\beta$ sheets. This peptide selectively binds to chloride channels in glioma cells and inhibits their migration and invasion (Dardevet et al., 2015). CTX can also interact with MMP-2 and Annexin A2 (ANXA2) that both are involved in invasion of glioma cells (Kesavan et al., 2009; Deshane et al., 2003). CTX and its analogs have received special attention for gliomas targeting (Xu et al., 2016; Cheng et al., 2014). In addition, CTX like peptides such as BmKCT and AaCTX isolated, respectively, from Buthus martinis' Karsch and Androctonus australis scorpion venoms show anti-glioma effects (Fau et al., 2007; Rjeibi et al., 2011). In silico studies on MelCT confirmed its interaction with $\mathrm{Cl}^{-}$channel and MMP-2 protein (Farsani et al., 2015; data are publishing). 
Most peptide toxins are produced by purification from the animal venoms or chemical synthesis that both are high-cost procedures. Recombinant production of toxins in Escherichia. coli is more economical and easier method. However, the small size of peptide toxins and high number of disulfide bonds cause low yield of recombinant toxins (Lyukmanova et al., 2010). There are some fusion tags that are able to enhance the solubility of peptide toxins and protect them from degradation (Esposito et al., 2006; Bogomolovas et al., 2009). In this study, we tried to produce recombinant MelCT in E.coli expression system by using a new designed vector based on $\mathrm{pET}$ vectors. The $\mathrm{pET} 32 \mathrm{Rh}$ vector was previously constructed in our laboratory to purify recombinant proteins easier and more cost-effective (Rezaei et al., 2021). To determine the anti-cancer function of recombinant MelCT, we investigated its effect on the proliferation and migration of glioma cells. To elucidate the molecular mechanisms involved in MelCT inhibitory effects, mRNA expression of MMP-2, ANXA2 and FOXM1 which are critically associated with glioma progression were investigated following rMeICT treatment.

MMP-2 overexpression is related to glioma malignancy and promotes glioma cell migration through degradation of ECM. Inhibition of MMP-2 expression significantly attenuates cell invasion (Roomi et al., 2007). Overexpression of ANX A2 has also been observed in glioma cells while it does not express in healthy brain tissue. ANXA2 expresses on surface of the cancer cells and upregulates ECM degradation via activation of pro-MMPs (Sharma, 2019). ANX A2 as a tumor-associated protein promotes cancer proliferation, migration, invasion, angiogenesis, inhibition of apoptosis and therapeutic resistance. The role of ANX A2 in cancer development makes this protein an interesting molecule for targeted therapy (Zhai et al., 2011).

FOXM1 belongs to the FOX (Forkhead Box) transcription factor family and is a key transcriptional regulator of the cell cycle. This factor is significantly involved in the tumorigenesis process of glioma (Cheng et al., 2013). Overexpression of FOXM1 is critical in many cancers and promotes proliferation, migration and invasion of glioma cells. Interestingly, FOXM1 has a binding site in the promoter of the MMP-2 gene and regulates its expression (Dai et al., 2007). Elevated FOXM1 level enhances glioma grade and invasion, whereas targeting of FOXM1 significantly suppresses the invasion of glioma cells (Zhang et al., 2017).

This study indicated that recombinant MelCT suppressed growth and migration of glioma cells and decreased the mRNA expression of ANX A2 and FOXM1 with no toxic effect on living organisms. These results suggest that MeICT can be considered as a new potential targeting agent for glioma therapy.

\section{Materials And Methods}

Animals and Cells

The U87-MG, human glioblastoma astrocytoma cell line, was kindly provided by Dr. Hossein Asadi from Mahan Research Institute, Kerman. Cells were grown in complete DMEM medium supplemented with $10 \%$ fetal bovine serum (FBS) and maintained in a humidified incubator with $5 \% \mathrm{CO} 2$ at $37^{\circ} \mathrm{C}$. The male BALB/c mice, weighing 20 to $30 \mathrm{~g}$, were purchased from Razi Animal Center, Tehran. The experimental 
animal procedures were performed in accordance with the National Institutes of Health guide for the care and use of Laboratory animals and by the Animal Ethics Committee of Shahrekord University.

Bioinformatics studies

The alignment of sequences was performed by ClustalW2 server (http://www.ebi.ac.uk/tools/msa/clustalw2/). DbD2 server (http://cptweb.cpt.wayne.edu/DbD2) was used to predict disulfide bond formation. Sequence homology analysis was performed by the NCBI BLAST server (http://www.ncbi.nlm.nih.gov/blast). The tertiary structure of MelCT was generated through Homology and Basic modeling by Modeler software. PDB structure of Insectotoxin I5a peptide with similar sequence to MeICT were selected as the template. The top 10 models of MeICT in PDB format were selected and the structure with the lowest molpdf energy was considered as the best proposed structure. Finally, it was examined by Cn3D software.

Cloning and expression of MelCT

The pET32Rh vector was used to clone of MelCT gene. This vector is a modified version of the pET32b plasmid that has lost His-tag sequence upstream of the multiple cloning sites. Therefore, the peptide containing C-terminal His-tag can be separated from thioredoxin part by two consecutive Ni-NTA purifications (Rezaei et al., 2021). The MelCT-encoding sequence and pET32Rh vector were digested by restriction endonucleases, $\mathrm{Bam} \mathrm{HI}$ and $\mathrm{Xho}$ I, and ligated with T4 ligase (Takara, Japan). The recombinant pET32Rh-MeICT vector was transformed into competent E.coli BL21 bacteria. Transformed bacteria were cultured in liquid LB medium up to $\mathrm{OD}_{600}=0.6$ and induced by $0.5 \mathrm{mM} \mathrm{IPTG}$ for $7 \mathrm{~h}$ at $28^{\circ}$ C. The bacteria were precipitated at $4000 \mathrm{rpm}$ for $15 \mathrm{~min}, 4^{\circ} \mathrm{C}$ and then $100 \mu \mathrm{l}$ of lysis buffer $(10 \mathrm{mM}$ Tris-

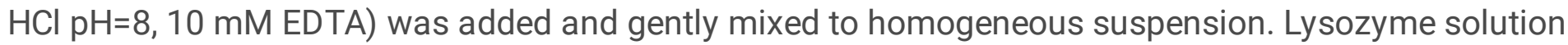
at a concentration of $1 \mathrm{mg} / \mathrm{ml}$ was added and placed on ice for $20 \mathrm{~min}$., the lysate was centrifuged at $12,000 \mathrm{rpm}$ for $20 \mathrm{~min}$ at $4^{\circ} \mathrm{C}$. The supernatant was analyzed on $12 \%$ SDS-PAGE and stained with Coomassie blue.

\section{Purification of recombinant rMelCT}

About $250 \mathrm{ml}$ media containing recombinant bacteria was cultured and induced for $7 \mathrm{~h}$ at $28^{\circ} \mathrm{C}$. Harvested cells were suspended in $4 \mathrm{ml}$ of lysis buffer containing $1 \mathrm{mM}$ imidazole, then sonicated and incubated on ice for $20 \mathrm{~min}$. The lysate was centrifuged and the supernatant was mixed with $1 \mathrm{ml}$ of NiNTA resin (Qiagen, Germany) and gently shacked for $1 \mathrm{~h}$ on ice. The mixture was loaded on an affinity column, washed with washing buffer (50 mM NaH2PO4, $300 \mathrm{mM} \mathrm{NaCl}$ and $20 \mathrm{mM}$ imidazole) and eluted two times with elution buffers containing $50 \mathrm{mM} \mathrm{NaH2PO4,} 300 \mathrm{mM} \mathrm{NaCl}, 250 \mathrm{mM}$ and $500 \mathrm{mM}$ imidazole, respectively. The eluted fraction was dialyzed against PBS solution overnight. Dialyzed samples containing recombinant Thioredoxin-MelCT (Trx-MelCT) were cleaved by $0.1 \mathrm{mg} / \mathrm{ml}$ enterokinase enzyme (Sigma, Germany) and analyzed on 12\% Tris-Tricine-SDS-PAGE. In order to purify rMeICT peptide from thioredoxin part, the peptide was re-purified by Ni-NTA column and dialyzed again. The rMeICT concentration was determined by Bradford assay. 
The growth and survival of glioma cells were measured using 3-(4, 5-dimethylthiazol-2-yl)-2, 5diphenyltetrazolium bromide (MTT) colorimetric assay. The cells were seeded at $5 \times 10^{3}$ density in 96 -well plate wells. The plate was incubated for $24 \mathrm{~h}$ and then treated with rMelCT concentrations of 1, 2, 3, 4, 5, and $6 \mu \mathrm{M}$ in triplicate. After $24 \mathrm{~h}, 20 \mu$ solution of $5 \mathrm{mg} / \mathrm{ml} \mathrm{MTT}$ (Sigma, Germany) in PBS was added and incubated at $37^{\circ} \mathrm{C}$ for $4 \mathrm{~h}$. The medium was discarded and $150 \mu \mathrm{l}$ of MTT solvent (containing dimethyl sulfoxide) was added to each well, plate was incubated for $30 \mathrm{~min}$. in the dark to dissolve formazan crystals. The absorbance was measured at $570 \mathrm{~nm}$. The percentage of living cells was calculated using: optical density (OD) value of treated cells/ OD value of untreated cells $\times 100$.

Wound healing assay

Cells $\left(2 \times 10^{5}\right)$ were seeded into a 12 -well plate until reaching confluence of $80-90 \%$, then were gently scratched using a $100 \mu \mathrm{l}$ pipette tip and washed carefully with PBS. New medium was added to the wells and cells were treated with 3 and $5 \mu \mathrm{M}$ concentration of rMelCT. Images were captured at 0,12 and $24 \mathrm{~h}$ and data were analyzed using ImageJ software $(\mathrm{NIH}, \mathrm{USA})$. Wound closure rate was calculated by placing the initial and final wound distance in the following equation: $100 \% \times[$ (initial -final)/initial $]$.

RNA extraction, cDNA synthesis and quantitative real time PCR

Cells were treated with $5 \mu \mathrm{M}$ rMelCT and harvested after $24 \mathrm{~h}$. Total RNA was extracted by TRIzol Reagent (Fermentas, USA). The cDNA was constructed using Prime Script RT Kit (Takara, Japan) according to the manufacturer's instructions. Quantitative real-time PCR with SYBR green method was used to evaluate the quantitative expression of ANXA2, FOXM1, and MMP-2 genes. The PCR was performed using following primers, ANXA2 forward: 5' CTGTGTGGTGGAGATGACTG3', ANEXA2 reverse: 5'CGGGGACTGTTATTCGCAAG3'; FOXM1 forward: 5'CAGTGGCTTCGAAAGATGAG3', FOXM1 reverse: 5'TCCTCAACCTTAACCTGTCG3'; MMP-2 forward: 5'CAGTGGCTTCGAAAGATGAG3', MMP-2 reverse: 5'TCCTCAACCTTAACCTGTCG3'. PCR condition consisted of $94^{\circ} \mathrm{C}$ for $20^{\prime \prime}$ followed by 40 cycles of $94^{\circ} \mathrm{C}$ for $3^{\prime \prime}$ and $60^{\circ} \mathrm{C}$ for $1 \mathrm{~min}$. Data were normalized to $\beta$-acting gene and relative quantities were calculated using $2^{-\Delta \Delta c t}$ equation.

In vivo toxicity

The in vivo toxicity of rMelCT was evaluated in mice by intraperitoneal injection. Different doses of toxin were injected in a range of $0.5 \mu \mathrm{g}$ to the increasing amount of $1,3,6$, and $10 \mu \mathrm{g}$ per $20 \mu \mathrm{g}$ body weight of mice. Five mice were used for each dose. Control mice were injected with PBS containing $0.1 \%(w / v)$ BSA solution.

\section{Results}

MeICT structure prediction 
Based on sequence homology, MelCT peptide was classified as a CTX-like peptide that can affect chloride channel (Fig.1A). The similarity of MelCT peptide with CTX, BmKCT and AaCTX was determined $81 \%, 82 \%$ and $68 \%$, respectively. Eight conserved cysteines in MelCT peptide form four disulfide bonds C1-C4, C2-C6, C3-C7 and C5-C8 that is consist with conventional pattern of $\mathrm{Cl}^{-}$channel toxins (Fig. 1B). The tertiary structure of MeICT was obtained by homology modeling using structure of Insectotoxin I5A templates. MeICT structure comprises an alpha helix formed by residues 11 to 20 and three $\beta$-sheets that one of them is located in the N-terminal and two of them are near the carboxyl terminal. The positions of the alpha helix and $\beta$-sheets are similar to CTX secondary structure (Fig.1C). However, because of the fewer number of residues; the length of MeICT $\beta$-strands is shorter than CTX.

\section{Expression and purification of MelCT peptide}

The gene encoding MeICT toxin was cloned into pET32Rh expression vector. The schematic structure of the recombinant MeICT fused to thioredoxin (Trx) with an enterokinase cleavage site in the N-terminal and a His-tag in the C-terminal has been shown in Fig. 2A. Recombinant Trx-MelCT with an expected molecular weight around $25 \mathrm{kDa}$ was observed in the soluble phase in SDS-PAGE (Fig. 2B). The rTrxMeICT was purified by Ni-NTA affinity chromatography (Fig. 2C) and cleaved using enterokinase enzyme. MelCT-His peptide was re-purified and detected around $4.5 \mathrm{kDa}$ on Tris-Tricine-SDS-PAGE (Fig. 2D). The amount of rMelCT-His peptide was determined about $0.6 \mu \mathrm{g} / \mu \mathrm{l}$ with Bradford assay.

Cytotoxicity assay on glioma cells

The effect of rMeICT on glioma cell proliferation and survival was analyzed using MTT test. The viability of cells was investigated in peptide concentrations of 1 to $6 \mu \mathrm{M}$. The results showed that rMelCT inhibits cell proliferation in a dose-dependent manner. The peptide concentration in which $50 \%$ of the cells are killed $\left(\mathrm{IC}_{50}\right)$ was determined approximately $3.8 \mu \mathrm{M}$ (Fig. $\left.3 \mathrm{~A}\right)$.

Wound healing assay

The effect of rMelCT on the migration of glioma cells was assessed by 3 and $5 \mu \mathrm{M}$ of the peptide. The ability of cells to fill the distance between cell boundaries depended on the dose of rMelCT (Fig. 3B). Compared with the control that was completely filled after $24 \mathrm{~h}$, the percentage of wound closure was determined about $58 \%$ and $22 \%$ when treated with 3 and $5 \mu \mathrm{M}$ of rMelCT, respectively (Fig 3C).

Evaluation of ANXA2, MMP-2 and FOXM1 gene expression

The effect of rIMeCT on expression of ANXA2, FOXM1 and MMP-2 genes in glioma cells was investigated by quantitative real-time PCR. Due to the significant role of these genes on cell migration, cells were treated with $5 \mu \mathrm{M}$ of $\mathrm{rMelCT}$ for 24 that was the concentration with high inhibition effect on migration. The mRNA expression levels of FOXM1 and ANXA2 were significantly decreased compared with the control by $77 \%$ and $98.6 \%$, respectively $(P<0.01)$ (Fig. 4). Despite the decrease in mRNA expression level of MMP-2, no statistically significant difference was determined. 
In vivo toxicity

The safety of rMelCT was evaluated by intraperitoneal injection of five doses of 0.5 to $10 \mu \mathrm{g}$ peptide in mice. The rMelCT did not induce any toxic symptoms after 24 hours and the following days. It suggests that this peptide is not toxic on mammals because the amount of $10 \mu \mathrm{g}$ is considered a high dose.

Statistical analysis

Data were analyzed using SPSS software. Student's t-test and one-way ANOVA were used to analyze the variances between two groups and more, respectively. A value of $P<0.05$ was considered statistically significant. Each experiment was repeated three times.

\section{Discussion}

Malignant glioma is the most aggressive brain tumor and does not respond to common therapeutic approaches. Increased attention has focused on CTX-like peptides and their analogs for targeting glioma cells (Xu et al., 2016; Cheng et al., 2014). Identification of new CTX-like peptides can introduce more efficient therapeutic agents and elucidate the molecular mechanisms of their function. In this study, recombinant MeICT, was produced by cloning into $\mathrm{PET} 32 \mathrm{Rh}$ vector. This vector facilitates the production of peptide using a two-step purification process and high-cost techniques such as HPLC (Highperformance liquid chromatography) do not need to the purification of recombinant protein (Rezaei, et al., 2021). Recombinant production of toxins is a challenging task due to the small size of toxins that results in peptide distraction in expression host and also their cysteine-rich structures that lead to protein aggregation. Although MelCT contains four disulfide bonds, its fusion with thioredoxin resulted in high expression level of the soluble peptide. The presence of thioredoxin in N-terminal of MeICT not only protects the peptide from degradation in E.coli, but also increases solubility of the protein by correct disulfide bond formation (LaVallie et al. 2000). In previous study on screening 13 different fusion partner for toxin expression in E.coli, the fusion to thioredoxin gave the highest yield of soluble toxin (Bogomolovas et al,. 2009). In this study we also obtained high yield of purified MelCT after two step purification. The anti-glioma activity of rMelCT-His demonstrated that His-tag in the C-terminal of peptide did not affect its function. It was consistent with a previous study that showed activation of recombinant His-CTX, although His-tag had been located in the N-ter of CTX (Deshane et al., 2003). It could be for the compact and specific structure of these peptides that is not affected by His-tag in $\mathrm{C}$ - or N-terminals.

In vivo toxicity of MeICT peptide was evaluated by peritoneal injection in mice. Due to the large number of blood vessels in this area, the peptide is rapidly introduced into the bloodstream. The high amount of MeICT did not induce any symptoms in mice that suggests this toxin can be an insect-specific peptide and does not affect normal mammalian cells.

The amino acid sequence of MelCT showed obvious homology to toxins specific for chloride channels. The interaction of MelCT with $\mathrm{Cl}^{-}$channel was determined by in silico studies (data are publishing). Our results demonstrated that MeICT inhibits glioma cell proliferation and migration in a dose-dependent 
manner. Previous studies have shown that CTX, BmKCT and AaCTX suppress growth and migration of glioma cells with different concentrations (Xu et al., 2016; Fau et al., 2007; Rjeibi et al., 2011). These differences might be related to how they interact with their targets. Although these toxins belong to the family of chloride channel toxins, they might also interact with other targets such as MMP-2 and ANXA2 (Deshane et al., 2003, Kesavan et al., 2009; Fu et al., 2011; Othman et al., 2017). It seems that the invasion of glioma cells is correlated with MMP-2 expression and secretion. Fu et al. showed that the alpha helix region of BmKCT interacts with the catalytic domain of MMP-2 and inhibits its function via $\mathrm{R}^{14}, \mathrm{R}^{17}$ and $\mathrm{K}^{15}$ residues (Fu et al., 2011). The interaction of MelCT with MMP-2 was investigated by molecular docking in our previous study and it was determined that the alpha helix region of MeICT peptide interacts with the catalytic domain of MMP-2 (Farsani et al., 2015). Comparison of the alpha helix region of CTX, BmKCT, MeICT and AaCTX showed that this region is relatively conserved in MeICT, CTX and BmKCT (Fig1A). Substitutions of residues with similar properties have occurred in this region, for example $\mathrm{R}^{14}$ in $\mathrm{CTX}$ and BmKCT peptides has been replaced by the $\mathrm{K}^{14}$ in MelCT that both are positively charged residues. In addition, $\mathrm{D}^{18}$ in CTX and MeICT has been replaced by $\mathrm{E}^{18}$ in BmKCT that both are negatively charged amino acids. This region is more distinctive in AaCTX, for example an uncharged residue, alanine, has been replaced in both positions 14 and 18. It has been suggested that the lower activity of AaCtx on migration of glioma cells is attributed to increasing the net charge of AaCTX to +4 , as well as the absence of negatively charged residues in alpha helix and N-terminal loop (Rjeibi et al., 2011). In MelCT peptide, the positive net charge has been determined +2 and the negatively charged amino acids $D^{18}$ and $D^{9}$ are present in the alpha-helix and $\mathrm{N}$-ter respectively. On the other hand, Othman et al. showed that residues 29-36 (8 C-ter residues) of CTX directly interact with MMP-2 (Othman et al., 2017). Furthermore, a recent study indicated that eight C-terminal residues of CTX are fundamental for its bioactivity and inhibition of cell migration (Dastpeyman et al., 2019). Seven C-ter residues of CTX and MelCT are identical and only $\mathrm{Y}^{29}$ in CTX has been replaced with $\mathrm{F}^{29}$ in MelCT, that both are aromatic residues with similar biochemical properties. Overall, MeICT displays more similarity with CTX than with BMKCT and AaCTX. The amino acid similarity of MeICT, AaCTX and BmKCT with CTX was determined $81 \%, 61 \%$ and $76 \%$, respectively. Therefore, it is possible that MeICT interacts with chloride channel and MMP-2 and inhibits progression of glioma cells, in similar ways like CTX.

Although the effective role of CTX and BmKCT in targeting glioma has been identified, the molecular mechanisms involved in this process have not been well described yet. In this study, we investigated the mRNA expression levels of ANXA2, MMP-2 and FOXM1 genes, which all are up-regulated in glioma cells and contribute to glioma migration and invasion. Previous studies have been shown that CTX and BMKCT significantly reduce MMP-2 protein secretion in glioma cells; however, no differences in MMP-2 mRNA expression were determined (El-Ghlban et al., 2014; Sun et al., 2017). Consistent with these reports, our study showed that MeICT does not affect the mRNA expression of MMP-2. Although, as mentioned above, MelCT may interact with MMP-2 and decrease the secretion of MMP-2 that was not investigated here. 
Although, ANXA2 protein was determined as a receptor for CTX, the roll of CTX on ANXA2 mRNA expression has not been investigated yet (Kesavan et al., 2009). In this study, we revealed the significant inhibitory effect of IMeCT on ANXA2 mRNA expression. ANXA2 is located in the pseudopodia of invasive glioma cells and binds to actin filaments of the cell membrane; therefore, its downregulation reduces cell migration (Zhai et al., 2011). Transcription of ANXA2 is regulated by external growth factors and kinase signaling pathways in cancer cells (Wang and Lin, 2014). How MeICT can mediate these molecular pathways requires further study.

Due to the direct association between overexpression of FOXM1 and glioma progression, the expression of FOXM1 was also investigated in this study (Cheng et al., 2013). Depletion of FOXM1 reduces glioma progression and it has been recognized as a therapeutic target for this cancer (Zhanget al., 2017, Halasi and Gartel, 2013). Here we reported down-regulation of FOXM1 mRNA level following MelCT treatment of glioma cells. Different mechanisms are involved in the regulation of FOXM1 that may contribute here (Liao et al., 2018). A previous report indicated that MMP-2 alters FOXM1 mRNA expression (Chetty et al., 2009). Therefore, MeICT may downregulate the expression of FOXM1 via interaction with MMP-2 and also decreasing MMP-2 secretion.

Both ANXA2 and FOXM1 mediate up-regulation of MMP-2 but by two distinct mechanisms (Sharma 2019; Dai et al., 2007). FOXM1 as a transcription factor binds to the MMP-2 promoter and increases its expression (Dai et al., 2007). Although FOXM1 was downregulated here, decrease in the MMP-2 mRNA level was not detected. It could be because of other regulation mechanisms that mediate MMP-2 transcription, for example different transcription factors bind to MMP-2 promoter including Sp1, YB-1, p53, and Stat3 (Pullen et al. 2018). On the other hand, ANXA2 regulates MMP-2 secretion via conversion of pro-MMP-2 to MMP-2. Studies have shown that downregulation of ANXA2 inhibits MMP-2 production and consequently inhibits invasion and angiogenesis of cancer cells (Zhao et al 2010). Both CTX and BMKCT decrease the protein level of MMP-2 that does not correlate with MMP-2 gene expression and probably is related to decrease in MMP-2 secretion (El-Ghlban et al., 2014; Sun et al., 2017). We suggest that the decrease in MMP-2 production can be caused by ANXA2 downregulation. Therefore, CTX-like peptides probably affect MMP-2 through two distinctive mechanisms; binding directly to MMP-2 and inhibiting its activation in addition to, suppressing indirectly MMP-2 production by down-regulation of ANXA2.

Based on different studies about function of CTX-like peptides, we concluded that these peptides contains MelCT might display anti-glioma activity through different mechanisms, including interaction with chloride channel, MMP-2 and ANXA2, downregulation of MMP-2 secretion and inhibition of ANXA2 and FOXM1 gene expression. The present study revealed that recombinant MelCT-His inhibits U87-MG glioma cell proliferation and migration. In vivo safety of MeICT and its potency to downregulate expression of cancer markers such as ANX A2 and FOXM1 can make it a promising factor for glioma targeting.

\section{Declarations}


Acknowledgements:

We thank Dr. Hossein Asadi for providing U87-MG cell line and Dr. Smayeh Reiisi for technical assistance. This study was supported by Shahrekord University.

\section{Author contributions}

Maryam Shahbazi Gandomkari: investigation, methodology, visualization roles, writing original draft. Hoda Ayat: supervision, conceptualization, project administration, validation, writing, review \& editing. Ali Mohammad Ahadi: data analysis, writing, review \& editing.

Conflict of Interest All authors declare they have no conflict of interest.

Ethical approval The permission for experimental animal procedures was obtained from Animal Ethics Committee of Shahrekord University

\section{References}

Bogomolovas J, Simon B, Sattler M, Stier G, (2009) Screening of fusion partners for high yield expression and purification of bioactive viscotoxins. Protein Expr Purif, 64:16-23.

Cheng SH, Tu Y, Zhang S (2013) FOXM1 Promotes Glioma Cells Progression by Up-Regulating Anxa1 Expression. PLoS ONE 8: 1-10.

Cheng Y, Zhao J, Qiao W, Chen K (2014) Recent advances in diagnosis and treatment of gliomas using chlorotoxin-based bioconjugates. Am. J. Nucl Med Mol 4: 385-405.

Chetty C, Bhoopathi P, Rao JS, Lakka S, (2009) Inhibition of Matrix Metalloproteinase-2 Enhances Radiosensitivity by Abrogating Radiation-induced FoxM1-mediated G2/M Arrest in A549 Lung Cancer Cells. Int J Cancer 124(10): 2468-2477.

Dai B, Kang SH, Gong W, et al., (2007) Aberrant FOXM1B expression increases matrix metalloproteinase-2 transcription and enhances the invasion of glioma cells. Oncogene 26: 6212-6219.

Dardevet L, Rani D, Abd El Aziz T, et al., (2015) Chlorotoxin: A Helpful Natural Scorpion Peptide to Diagnose Glioma and Fight Tumor Invasion. Toxins 7: 1079-1101.

Dastpeyman M, Giacomin P, Wilson D, et al, (2019) A C-Terminal Fragment of Chlorotoxin Retains Bioactivity and Inhibits Cell Migration. Front Pharmacol 10 (250): 1- 10.

Deshane J, Garner CC, Sontheimer H, (2003) Chlorotoxin inhibits glioma cell invasion via matrix metalloproteinase-2. J Biol Chem 278: 4135-44.

El-Ghlban S, Kasai T, Shigehiro T, et al, (2014) Chlorotoxin-Fc Fusion Inhibits Release of MMP-2 from Pancreatic Cancer Cells. BioMed Res Int 1: 1-10. 
Esposito D, Chatterjee DK, (2006) Enhancement of soluble protein expression through the use of fusion tags. Currt Opin Biotechnol, 17:353-358

Farsani FM, Ayat H, Ahadi AM, (2015) Molecular Modeling and Docking Studies on the First ChlorotoxinLike Peptide from Iranian Scorpion Mesobuthus eupeus (Meict) and SNP Variants of Matrix Methaloproteinase-2 (MMP-2). Iranain J Toxicol 9: 1368-1376.

Fau YJ, Yin LT, Liang AH, et al, (2007) Therapeutic potential of chlorotoxin-like neurotoxin from the Chinese scorpion for human gliomas. Neurosci Lett 412: 62-67.

Fu YJ, An N, Chan KG, et al, (2011) A model of BmK CT in inhibiting glioma cell migration via matrix metalloproteinase-2 from experimental and molecular dynamics simulation study. Biotechnol Lett 33: $1309-1317$.

Halasi M, Gartel AL, (2013) Targeting FOXM1 in cancer. Biochem Pharmacol 85: 644-652.

Ilkhanizade S, Ayat H, Ahadi AM, Pirali K, (2011) Sequencing and comparative-bioinformatic analysis of chlorotoxin-like peptide from Iranian scorpion Mesobuthus eupeus. J SKU Med Sci. 13: 27-36.

LaVallie ER, Lu Z, Diblasio-Smith EA, Collins-Racie LA, McCoy JM (2000) Thioredoxin as a fusion partner for production of soluble recombinant proteins in Escherichia coli. Methods Enzymol 326: 322-340.

Liang J, Lv X, Lu C, et al, (2020) Prognostic factors of patients with Gliomas - an analysis on 335 patients with Glioblastoma and other forms of Gliomas, BMC cancer 20: 35, 1-7.

Liao G, Li X, Zeng S, Liu C, Yang S, Yang L, Hu C, Bai J, (2018) Regulation of the master regulator FOXM1 in cancer. J Cell Commun Signal 16:57, 1-15.

Lyukmanova EN, Shulepko MA, Shenkarev ZO, Dolgikh DA, Kirpichnikov MP, (2010) In vitro production of three-finger neurotoxins from snake venoms, a disulfide rich proteins. Problems and their solutions (Review). Russ J Bioorg Chem, 36:137-145.

Kesavan K, Ratliff J, Johnson EW, et al, (2009) Annexin A2 is a molecular target for TM601, a peptide with tumor-targeting and anti-angiogenic effects. J Biol Chem 285: 4366-4374.

Othman $\mathrm{H}$, Wieninger SA, ElAyeb M, et al, (2017) In silico prediction of the molecular basis of CITx and AaCTx interaction with matrix metalloproteinase-2 (MMP-2) to inhibit glioma cell invasion. J Biomol Struct Dyn 35: 2815-2829. doi: 10.1080/07391102.2016.1231633

Pullen NA, Pickford AR, Perry MM, et al, (2018) Current insights into matrix metalloproteinases and glioma progression: transcending the degradation boundary. Metalloproteinases In Med, 5: 13-30

Rezaei L, Ayat $\mathrm{H}$, Ahadi AM, (2021) Design and construction of pET32b(+) Rh expression vector based on pET system to facilitate purification. $J$ cell mol res. 34: (2), 169-181. 
Rjeibi K, Mabrouk H, Mosrati C, et al, (2011) Purification, synthesis and characterization of AaCTX, the first chlorotoxin-like peptide from Androctonus australis scorpion venom, Peptides 32: 656-663.

Roomi MW, Ivanov V, Kalinovsky T, et al, (2007) Inhibition of glioma cell line A-172 MMP activity and cell invasion in vitro by a nutrient mixture. Med Oncol 24: 231-8.

Sharma MC, (2019) Annexin A2 (ANNEXIN A2): An emerging biomarker and potential therapeutic target for aggressive cancers, Int J Cancer 144: 2074-2081.

Sun N, Zhao L, Qiao W, et al, (2017) BmK CT and I-BmK CT suppress the invasion of glioma cells in vitro via matrix metalloproteinase-2, Mol Med Rep 15: 2703-2708.

Turner KL, Sontheimer $\mathrm{H}(2014) \mathrm{Cl}^{-}$and $\mathrm{K}^{+}$channels and their role in primary brain tumour biology, Philos Trans R Soc B 369: 1-9.

Wang CY, Lin CF (2014) Annexin A2: Its Molecular Regulation and Cellular Expression in Cancer Development Dis Markers 1-10.

Xu T, Fan Z, Li W, et al. (2016). Identification of two novel Chlorotoxin derivatives CA4 and CTX-23 with chemotherapeutic and anti-angiogenic potential. Sci Rep 25: 1-14.

Zhai H, Acharya S, Gravanis I, et al. (2011) Annexin A2 Promotes Glioma Cell Invasion and Tumor Progression. J Neurosci. 31(40): 14346-60.

Zhang X, LV Q, Huang Y, et al. (2017) Akt/FoxM1 signaling pathway-mediated upregulation of MYBL2 promotes progression of human glioma. J Exp Clin Cancer Res 36: 1-18.

Zhao P, Zhang W, Tang J, et al. (2010) Annexin Il promotes invasion and migration of human hepatocellular carcinoma cells in vitro via its interaction with HAb18G/CD147. Cancer Sci 101: 387-395.

\section{Figures}


MeICT MCMPCFTTDH

CTX MCMPCFTTDH

BmKCT MCGPCFT TDA

AaCTX MCIPCFTTNP

Consensus

MCMPCFTTDH NMARKCNDCC GGXGRGKCFG PQCLCR - -

Conservation

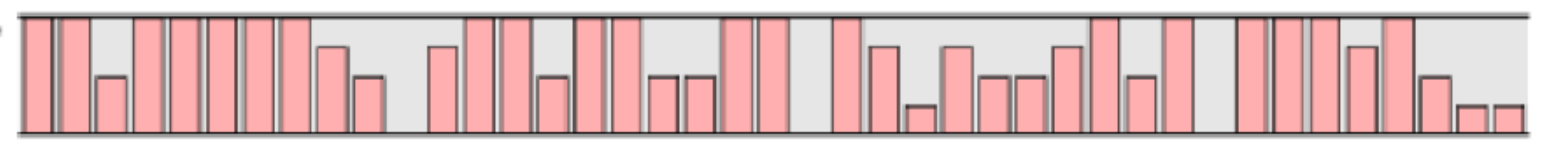

B

MCMPCFTTDHNMAKKCNDCCGGYGKCFGPQCLCR

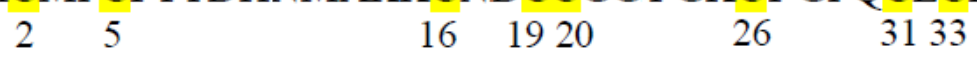

C

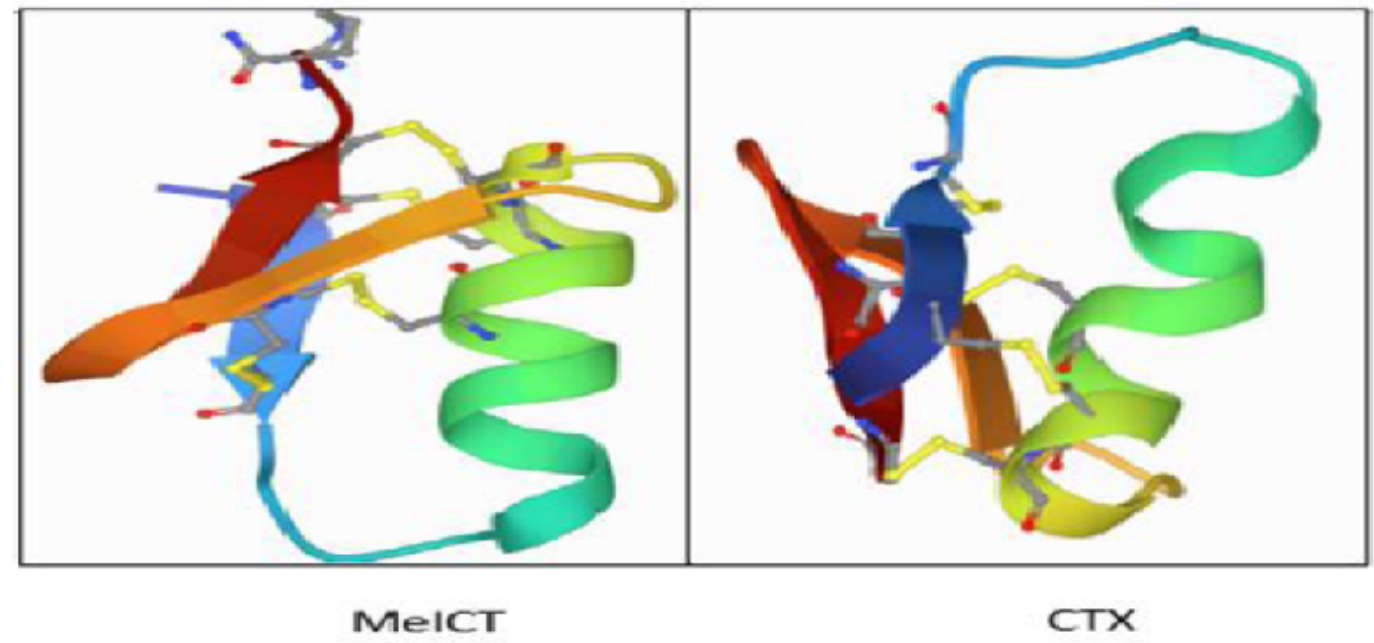

Figure 1

Amino acid sequence and structure of MelCT. (A) Comparison of MeICT, CTX, BmKCT and AaCTX peptide sequences in CLC software. The conserved cysteine residues is highlighted with yellow. Secondary structurally elements are marked by arrows as $\beta$-sheets and helix. (B) The cysteines are numbered and disulfide bonds are displayed using lines. (C) Comparison of the predicted 3D structures for MeICT and chlorotoxin. The beta sheets are shown in arrows, alpha helix in green and disulfide bridge in yellow. 
A
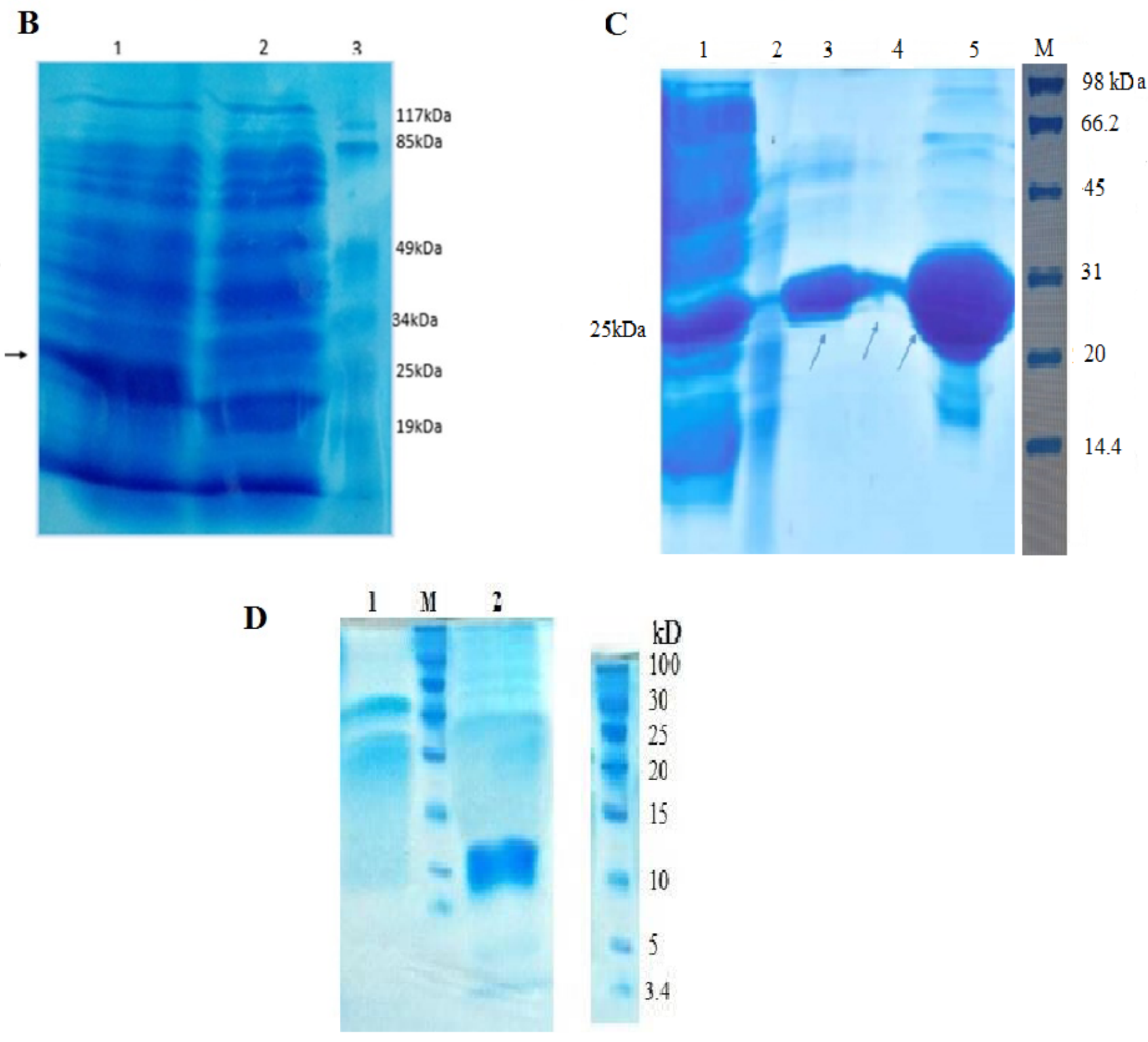

\section{Figure 2}

Preparation and purification of MelCT. (A) Schematic of construct encoding rTrx-MelCT fusion peptide. Cleavage site of enterokinase was shown in green. (B) Expression of rTrx-MelCT was determined by SDSPAGE analysis. Lane 1: soluble expression after induction with IPTG; Lane 2: insoluble expression after induction. Lane 3: protein size marker. (C) Purification of rTrx-MeICT was analyzed by SDS-PAGE, Lane 1: pre-purification protein sample, Lane 2: protein sample before induction; Lane 3-6: purified rTrx-MelCT by 250 and $500 \mathrm{mM}$ of imidazole, respectively. (D) Purification of rMeICT was analyzed by SDS-PAGE. Lane 
1: rTrx-MelCT after cleavage with enterokinase enzyme; Lane 2: protein size marker; Lane 3: purified rMelCT around $4.5 \mathrm{kDa}$.
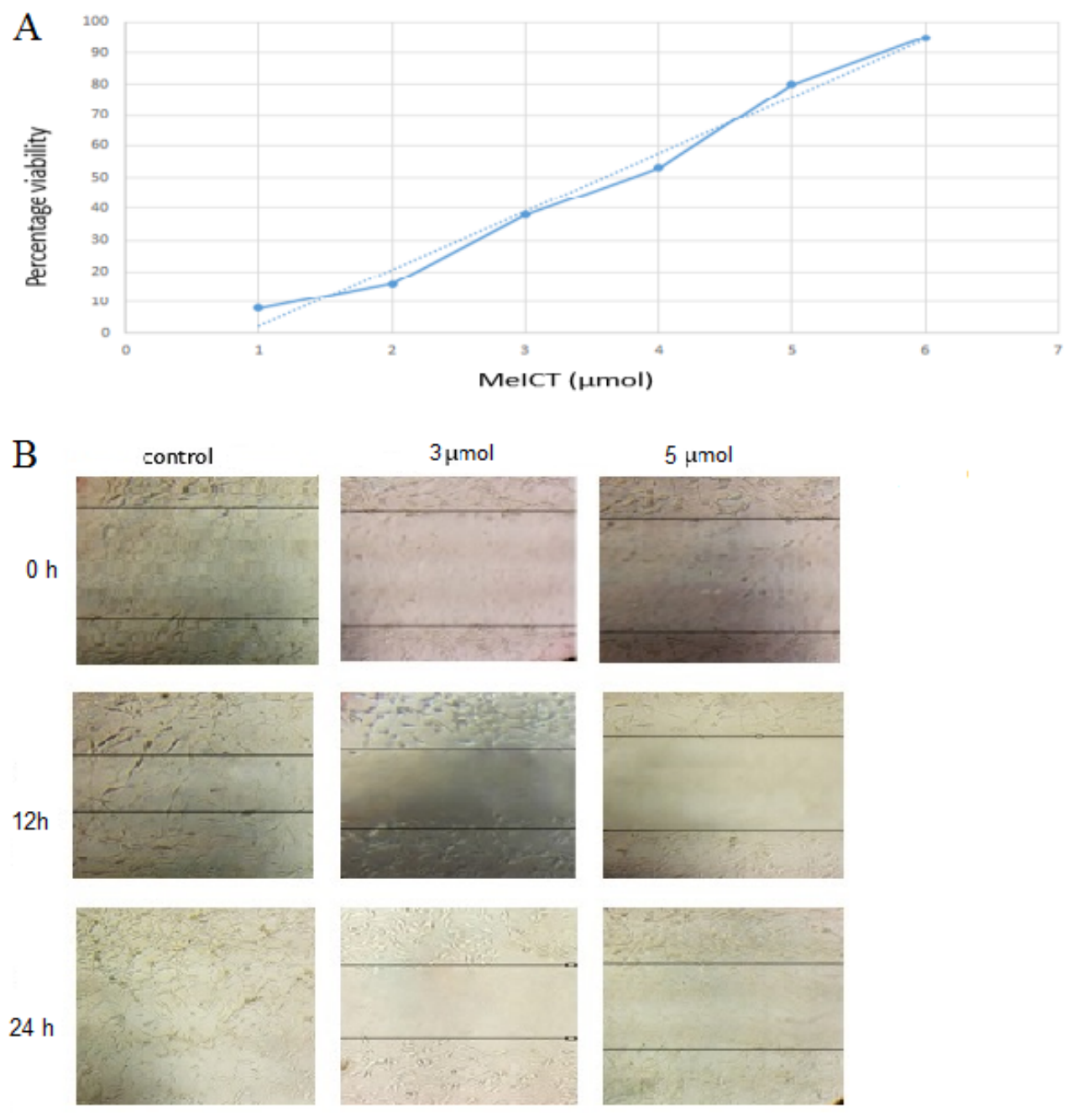

$\mathrm{C}$

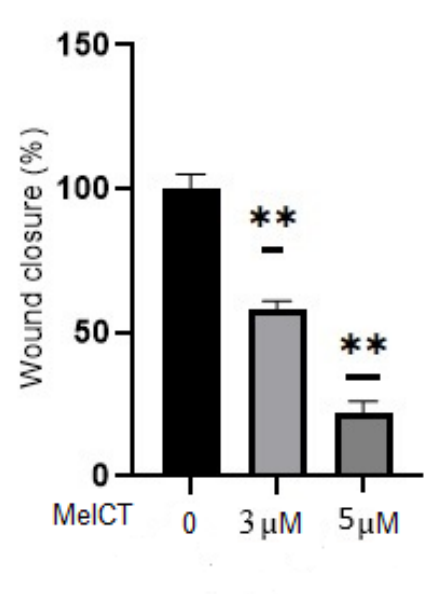

Figure 3

Effect of IMeCT on U87-MG cells. (A) Proliferation of cells was inhibited by IMeCT dose-dependently. (B) Migration of cells following treatment with $3 \mu$ and $5 \mu \mathrm{M}$ of MelCT was determined by wound-healing assay. (C) Quantification of wound closure in control and treated cells at $0 \mathrm{~h}, 12 \mathrm{~h}$ and $24 \mathrm{~h}$. ${ }^{* * \mathrm{P}}<0.01$ 

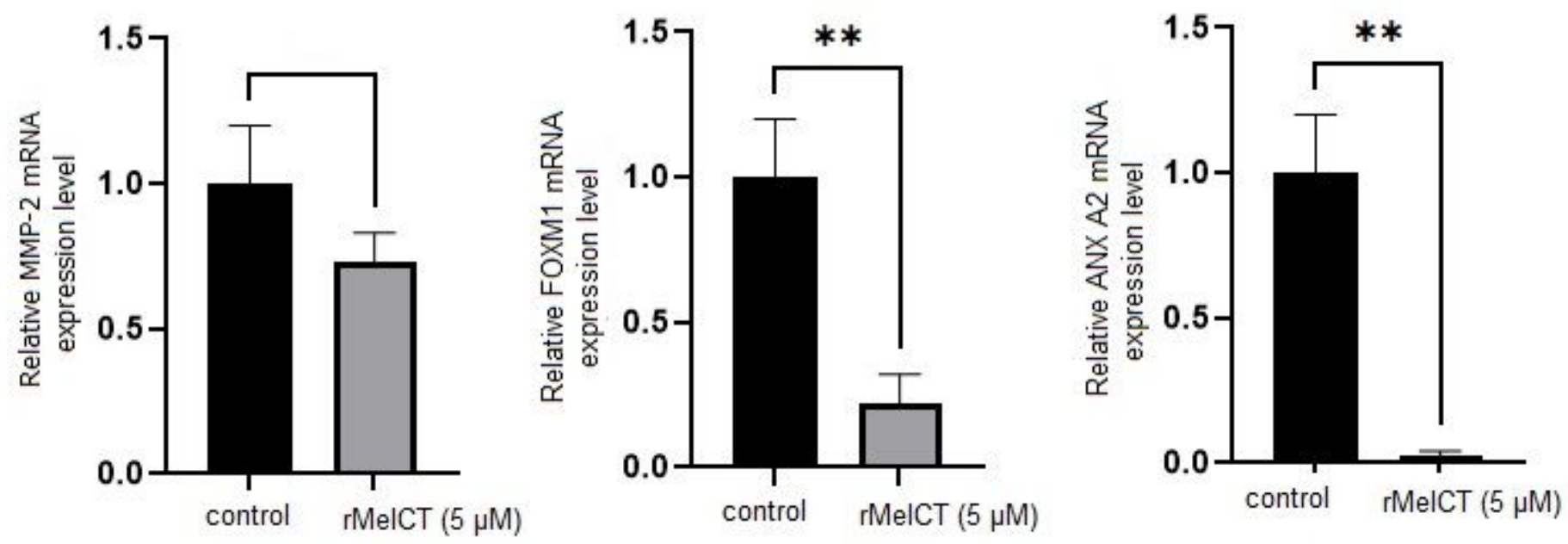

Figure 4

MelCT significantly decreased the mRNA expression of ANXA2 and FOXM1. U87-MG cells were treated with $5 \mu \mathrm{M}$ of MelCT and mRNA expression levels of ANXA2, MMP-2 and FOXM1 detected by RT-qPCR. $\star \star P<0.01$ 